\title{
The Fabrication of THz Emitting Mesas by Reactive Ion-Beam Etching of Superconducting Bi2212 with Multilayer Masks
}

\author{
H. Koseoglu • F. Turkoglu • Y. Simsek · L. Ozyuzer
}

Received: 5 September 2010 / Accepted: 6 September 2010 / Published online: 28 September 2010

(C) Springer Science+Business Media, LLC 2010

\begin{abstract}
Generation of powerful $\mathrm{THz}$ radiation from intrinsic Josephson Junctions (IJJs) of $\mathrm{Bi}_{2} \mathrm{Sr}_{2} \mathrm{CaCu}_{2} \mathrm{O}_{8+\delta}$ (Bi2212) may require mesas with large lateral dimensions. However, there are difficulties in fabrication of perfect rectangular mesas. The lateral angles of mesas should be close to 90 degrees to obtain IJJs with same planar dimensions for synchronization of IJJs. We patterned Ta/photoresist and photoresist/Ta/photoresist masks on Bi2212 and used selective ion etching to overcome the thick photoresist layer shading on the lateral dimension of mesa during the ion-beam etching. The reactive ion-beam etchings have been done with ion beams of $\mathrm{Ar}, \mathrm{N}_{2}$ and $\mathrm{O}_{2}$, and we have obtained mesas about $1 \mu \mathrm{m}$ with lateral angle of approximately 50 to $75^{\circ}$, which is better than the mesas fabricated with photoresist mask.
\end{abstract}

Keywords Reactive ion-beam etching - Intrinsic Josephson junctions · Terahertz radiation · Bi2212

\section{Introduction}

Terahertz $(\mathrm{THz})$ radiation is a part of the electromagnetic spectrum, lying between microwaves and far-IR. This region covers frequencies ranging from $0.1-10 \mathrm{THz}$ and wavelengths from $3 \mathrm{~mm}$ to $0.03 \mathrm{~mm}$. Recently, the research community of science and technology have become interested in the electromagnetic waves in terahertz frequency because

H. Koseoglu ( $\varangle) \cdot$ F. Turkoglu · Y. Simsek · L. Ozyuzer Department of Physics, Izmir Institute of Technology, 35430 Izmir, Turkey

e-mail: hasankoseoglu@iyte.edu.tr of their emerging application areas, including physics, biology, chemistry, astronomy, medicine etc. [1]. Electromagnetic waves with frequencies below and above the $\mathrm{THz}$ frequency range are often generated by semiconducting electronics based on high-speed transistors and the photonics based on the semiconducting lasers, respectively. However, there is still lack of $\mathrm{THz}$ wave sources and there are difficulties in their generation. On the other hand, their functional advantages in many application areas entail the evolution of the $\mathrm{THz}$ sources in science [2].

The research on realizing $\mathrm{THz}$ wave sources with high power, low cost and portability has been increasing. Since they are planned to be used in many areas, they should be continuous, coherent and frequency tunable as well. Therefore, the research has gone toward the novel $\mathrm{THz}$ sources, which include layered structures of high-temperature superconductors (HTSs). One of the several reasons why HTSs may be a suitable candidate for the generation of $\mathrm{THz}$ radiation is their layered structure, which enables the propagation of electromagnetic wave by unique excitation called Josephson plasma oscillations, and the frequency of the Josephson radiation is in the $\mathrm{THz}$ range [3].

High-temperature superconducting $\mathrm{Bi}_{2} \mathrm{Sr}_{2} \mathrm{CaCu}_{2} \mathrm{O}_{8+\delta}$ (Bi2212) single crystal has natural stacked junctions called intrinsic Josephson junctions (IJJs) [4]. It has a large energy gap and wide doping range [5], which is suitable for the $\mathrm{THz}$ region. So it may play an important role for generation of $\mathrm{THz}$ radiation. When an external current is applied along the $c$-axis of Bi2212 crystal, the ac Josephson current in the resistive state excites a cavity resonance mode of a Josephson plasma wave in the sample. The excited standing wave of the Josephson plasma is converted to a terahertz electromagnetic wave at the mesa surfaces and the electromagnetic wave is emitted into the vacuum space (Fig. 1) [6]. Generation of powerful $\mathrm{THz}$ radiation requires mesas with 
Fig. 1 The schematic of mesa and electrical contacts on Bi2212 for emission of $\mathrm{THz}$ radiation

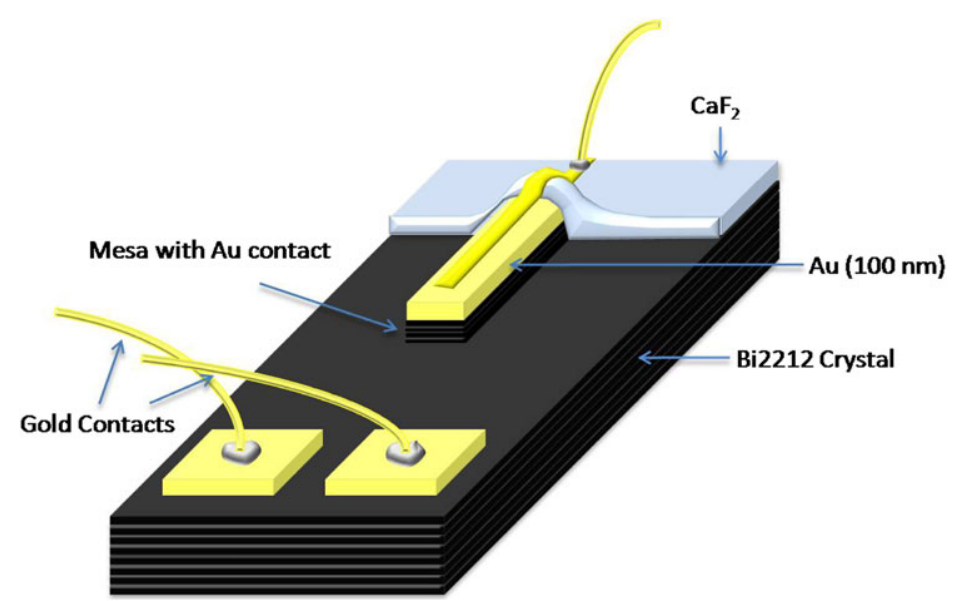

large lateral dimension, but there are difficulties in fabrication of perfect rectangular mesas. It should be close to 90 degrees to obtain IJJs with the same planar dimensions for synchronization of all IJJs at the same frequency [7].

Both experimental and theoretical studies have been achieved for the high power emission of $\mathrm{THz}$ waves from Bi2212 [8]. However, the details of the mechanism have not been clarified yet. It is important to design mesa structures with perfect lateral angles to shed light on the effect of edges on powerful $\mathrm{THz}$ emission. In this study, new multilayer masking techniques have been explored for selective etching of layered superconductor Bi2212.

\section{Experiment}

The single crystals of Bi2212 used in our studies have been prepared by D.G. Hinks at Argonne National Laboratory using the traveling solvent floating zone method. We also grow Bi2212 single crystals by the self-flux method in our institute. Bi2212 single crystal with the size of $1 \times 1 \mathrm{~mm}^{2}$ from smooth $a-b$ surface is glued onto a sapphire substrate by silver epoxy. In order to get a fresh and smooth surface on Bi2212, the crystal was mechanically cleaved with scotch tape. After the cleaving process, a gold layer with thickness of 50 to $100 \mathrm{~nm}$ is immediately deposited by thermal evaporation technique on the fresh surface of the crystal in order not to lose clean fresh surface. In this work, we used three different masks to fabricate mesa. They are a single-layer photoresist (PR) mask and two different multilayer masks, which are $\mathrm{Ta} / \mathrm{PR}$ and $\mathrm{PR} / \mathrm{Ta} / \mathrm{PR}$ masks. In all three masks, all experimental steps are the same until the gold evaporation. After the gold evaporation, the experimental steps have different procedures and etching recipes. The mesas with rectangular surface areas, $100 \times 300 \mu \mathrm{m}^{2}$ to $60 \times 300 \mu \mathrm{m}^{2}$, were prepared in this study.

Tantalum metal was deposited on $\mathrm{Au}$ by dc magnetron sputtering for $\mathrm{Ta} / \mathrm{PR}$ masks [9]. To obtain PR/Ta/PR mask, firstly photoresist was covered onto the gold layer with spin

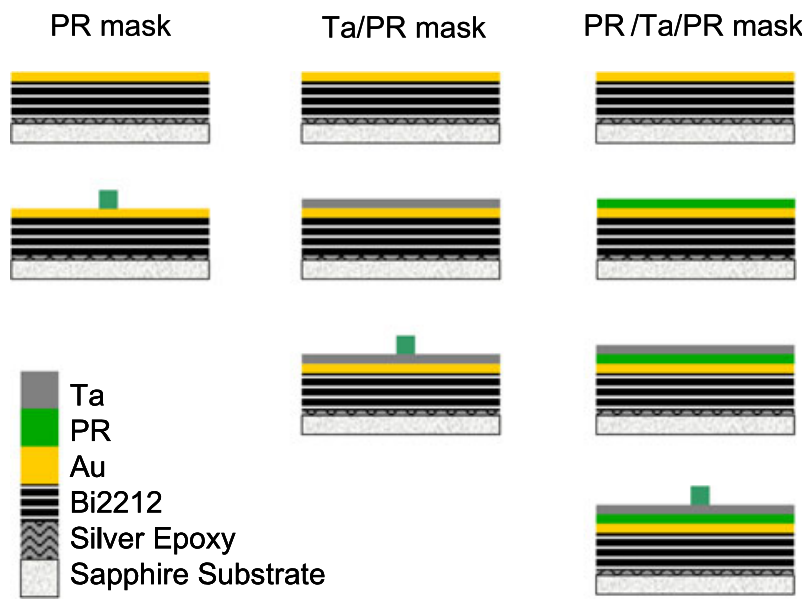

Fig. 2 Mesa fabrication processes for three different masking techniques. The samples are ready for reactive ion-beam etching

coater, having a 750-nm layer of resist, and then hard baked in an oven at the $200^{\circ} \mathrm{C}$ for 40 minutes to get strongest photoresist layer. After that, Ta metal was deposited onto the PR layer. The aim of Ta/PR and PR/Ta/PR masks is to form a mesa with another material, apart from the PR, which can be used for selective etching [10]. Since the thickness of photoresist is usually high and its edges are not perpendicular to the crystal, the edges of an etched shape are like the curvature edges of mesa. Hence the right angle rectangular shape cannot be obtained. Furthermore, the photoresist can be etched by Ar ions rapidly and tall mesas cannot be realized.

The final step in mesa fabrication is the standard optical photolithography which is used in micro fabrication to pattern a photoresist layer on Au and Ta layers of crystals. Thus selective areas without regions protected by PR layer are etched down by ion beam, while PR patterns protect mesa areas from etching. Photolithography includes several steps in sequence shown in the literature. The schematic of mesa fabrication processes up to reactive ion-beam etching is shown in Fig. 2. 
After the preparation of the mesa shaped PR pattern, the sample was mounted into the ion-beam etching system that produces an ion beam accelerated through the surface of the sample to etch down the area unprotected by PR layer on crystal. For all three masks, different etching recipes were used. For the single-layer mask, an ion beam etching process was applied to the mounted sample (Bi2212/Au/PR) with

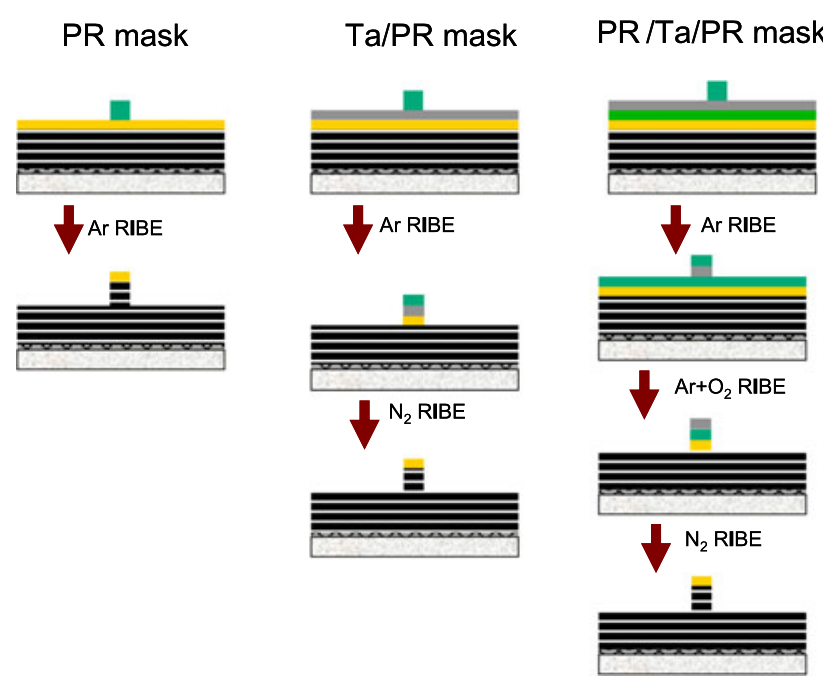

Fig. 3 Schematic views of the samples before and after the etching process

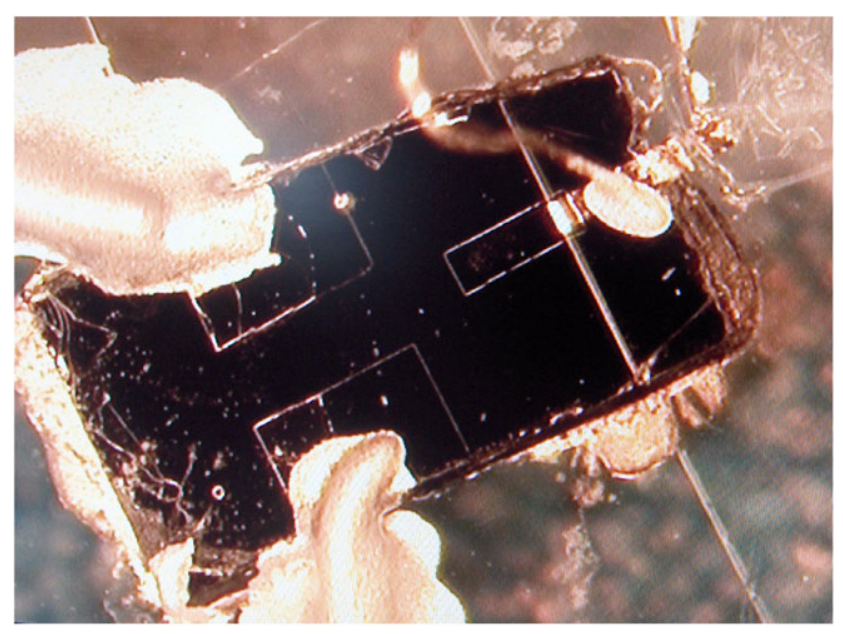

Fig. 4 The electrical measurement configuration of Bi2212 mesa
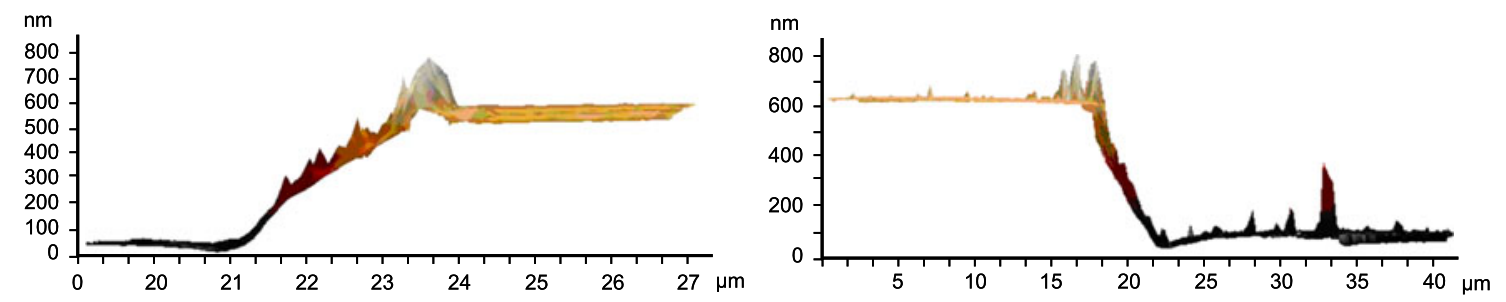

Ar plasma until the PR layer onto the Au layer of mesa was also removed. For the Ta/PR multilayer mask, a reactive ion-beam etching (RIBE) process was applied to the mounted sample (Bi2212/Au/Ta/PR) with Ar plasma until the $\mathrm{Au}$ and Ta was removed, then using $\mathrm{N}_{2}$ plasma, etching of Bi2212 was continued to get the Bi2212 mesa until the $\mathrm{Ta}$ layer onto the Au layer of mesa was removed. We used $\mathrm{N}_{2}$ plasma for the etching of Bi2212, because the etching rate of Ta is smaller than Bi2212. This enables us to fabricate taller $(\approx 1 \mu \mathrm{m})$ and high lateral angle mesas. For the $\mathrm{PR} / \mathrm{Ta} / \mathrm{PR}$ multilayer mask, a RIBE process was applied to the mounted sample (Bi2212/Au/PR/Ta/PR) with Ar plasma until the Ta and Au layers out of the mesa are removed. Then the first PR layer onto the outside of mesa was removed with $\mathrm{Ar}+\mathrm{O}_{2}$ plasma and then etching of $\mathrm{Bi} 2212$ with $\mathrm{N}_{2}$ plasma was continued to get the Bi2212 mesa until the Ta layer onto the PR layer of mesa was removed. After the Ta layer was etched, the mesa was tall with high lateral angle. There were PR layers at the top of the mesa. To etch the first PR layer, etching was ended with $\mathrm{Ar}+\mathrm{O}_{2}$ plasma until the PR layer onto the Au layer of mesa was etched. Figure 3 shows gas recipes for three RIBE processes.

After the mesa fabrication, in order to establish electrical contact to the gold layer on top of the mesa, $\mathrm{CaF}_{2}$ layer was evaporated through a shadow mask onto the top part of the crystal. Subsequently, a gold stripe was evaporated through another shadow mask onto the mesa and the $\mathrm{CaF}_{2}$. Finally, gold wires were attached to the strip over the $\mathrm{CaF}_{2}$ and two contact pads (Fig. 4). The surface (lateral dimensions) characterizations and electrical characteristics of the mesas were obtained.

\section{Results}

Figures 5, 6 and 7 show the atomic force microscopy (AFM) image of two long sides (called edge 1 and 3 ) of three mesas obtained by three different multilayer layer masking technique. The AFM analysis results of the fabricated mesas can be seen in Table 1. It can be seen from Table 1 that using a multilayer masking technique instead of a single layer indicates a distinctive increase of the lateral angle, and this is proof of the approaching of a nearly rectangular mesa.

Fig. 5 Side view of AFM measurement of Mesa\#1 (Bi2212/Au) 

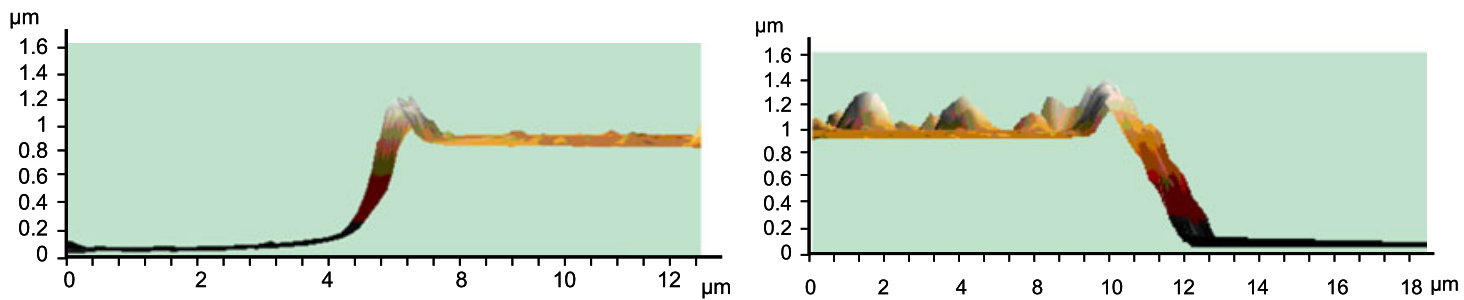

Fig. 6 Side view of AFM measurement of Mesa\#2 (Bi2212/Au/Ta)
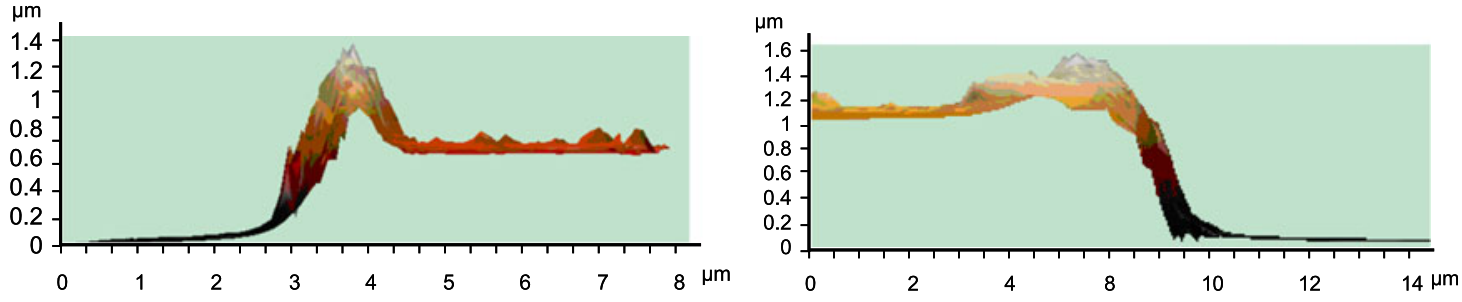

Fig. 7 Side view of AFM measurement of Mesa\#3 (Bi2212/Au/PR/Ta)

Table 1 AFM analysis results of fabricated mesas

\begin{tabular}{lll}
\hline Mesa name and mask & Edge 1 & Edge 3 \\
\hline Mesa\#1 (Bi2212/Au) & $570 \mathrm{~nm}-12^{\circ}$ & $650 \mathrm{~nm}-13^{\circ}$ \\
Mesa\#2 (Bi2212/Au/Ta) & $860 \mathrm{~nm}-51^{\circ}$ & $870 \mathrm{~nm}-52^{\circ}$ \\
Mesa\#3 (Bi2212/Au/PR/Ta) & $860 \mathrm{~nm}-75^{\circ}$ & $770 \mathrm{~nm}-35^{\circ}$ \\
Mesa\#4 (Bi2212/Au/PR/Ta) & $850 \mathrm{~nm}-65^{\circ}$ & $810 \mathrm{~nm}-45^{\circ}$ \\
Mesa\#5 (Bi2212/Au/PR/Ta) & $830 \mathrm{~nm}-58^{\circ}$ & $580 \mathrm{~nm}-45^{\circ}$ \\
Mesa\#6 (Bi2212/Au/PR/Ta) & $930 \mathrm{~nm}-50^{\circ}$ & $820 \mathrm{~nm}-42^{\circ}$ \\
Mesa\#7 (Bi2212/Au/PR/Ta) & $778 \mathrm{~nm}-60^{\circ}$ & NA \\
Mesa\#8 (Bi2212/Au/PR/Ta) & $705 \mathrm{~nm}-71^{\circ}$ & NA \\
Mesa\#9 (Bi2212/Au/PR/Ta) & $791 \mathrm{~nm}-70^{\circ}$ & NA \\
\hline
\end{tabular}

An increase of the lateral angle of approximately 50 to $75^{\circ}$ is better than the previous studies. This is the most important achievement of the present study. Since the ion beam with incident angle of $22.5^{\circ}-67.5^{\circ}$ is accelerated through the sample holder, differences in lateral angles of mesa may occur. If we rotate the sample holder just in one direction, height and lateral angle differences between the edges of the mesa exists.

\section{Conclusion}

In this study, three different masking techniques have been used to fabricate Bi2212 mesas for THz emission. It is found that multilayer $\mathrm{PR} / \mathrm{Ta} / \mathrm{PR}$ masks can produce a steep mesa edge as high as lateral angles of 71 degrees. This will allow us to generate $\mathrm{THz}$ emission from mesas with high power, which will include all mesas emitting with single frequency. This is because it has already been shown that the mesa width determines the $\mathrm{THz}$ emission frequency of mesa.
Acknowledgements This research is supported in part by the TUBITAK (Scientific and Technical Council of Turkey) project No. $108 \mathrm{~T} 238$

\section{References}

1. Tonouchi, M.: Cutting-edge terahertz technology. Nat. Photonics 1, 97-105 (2007)

2. Bae, M.H., Lee, H.C.: Progress in THz generation using Josephson fluxon dynamics in intrinsic junctions. IEICE Trans. Electron. E 89, 411 (2006)

3. Tachiki, M., Koyama, T., Takahashi, S.: Electromagnetic Phenomena Related to a low frequency plasma in cuprate superconductors. Phys. Rev. B 50, 7065 (1994)

4. Kleiner, R., Steinmeyer, F., Kunkel, G., Müller, P.: Intrinsic Josephson effects in $\mathrm{Bi}_{2} \mathrm{Sr}_{2} \mathrm{CaCu}_{2} \mathrm{O}_{8}$ single crystals. Phys. Rev. Lett. 68, 2394 (1992)

5. Ozyuzer, L., Zasadzinski, J.F., Miyakawa, N.: Tunneling spectra and superconducting gap in $\mathrm{Bi}_{2} \mathrm{Sr}_{2} \mathrm{CaCu}_{2} \mathrm{O}_{8+d}$ and $\mathrm{Tl}_{2} \mathrm{Ba}_{2} \mathrm{CuO}_{6+d}$. Int. J. Mod. Phys. B 13, 3721 (1999)

6. Ozyuzer, L., Koshelev, A.E., Kurter, C., Gopalsami, N., Li, Q., Tachiki, M., Kadowaki, K., Tamamoto, T., Minami, H., Yamaguchi, H., Tachiki, T., Gray, K.E., Kwok, W.K., Welp, U.: Emission of coherent THz radiation from superconductors. Science 318, 1291 (2007)

7. Ozyuzer, L., Simsek, Y., Koseoglu, H., Turkoglu, F., Kurter, C., Welp, U., Koshelev, A.E., Gray, K.E., Kwok, W.K., Yamamoto, T., Kadowaki, K., Koval, Y., Wang, H.B., Muller, P.: Terahertz wave emission from intrinsic Josephson junctions in high-Tc superconductors. Supercond. Sci. Technol. 22, 114009 (2009)

8. Hu, X., Lin, S.Z.: Supercond. Sci. Technol. 23, 053001 (2010)

9. Tuna, O., Selamet, Y., Aygun, G., Ozyuzer, L.: High quality ITO thin films grown by DC and RF sputtering without oxygen. J. Phys. D, Appl. Phys. 43, 055402 (2010)

10. Nagai, Y., Tsuru, K., Terada, A., Yanagisawa, K.: Micropatterning of high-Tc superconducting film by nitrogen ion beam and Ta/resist multilayer mask. IEEE Trans. Magn. 27, 1622 (1991) 